\title{
Strange Attractors and Human Interaction: Leading Complex Organizations through the Use of Metaphors
}

\author{
DONALD L. GILSTRAP \\ Southwestern Oklahoma State University (USA)
}

\begin{abstract}
This article is intended to explore the theoretical background behind complexity science in management and leadership and provide ways to approach educational leadership research through the use of strange attractor metaphors. Historical and contemporary leadership strategies have incorporated modernistic models that sometimes perpetuate problematic aspects of educational management rather than provide progressive solutions. Several leadership researchers have shown, however, there is tremendous potential for the emergent properties of complexity theory in organizational dynamics. The recognition and utilization of strange attractors as metaphorical constructs of chaos theory also provide us with an elaboration of teaching and educational leadership theory. Strange attractors seem to exist metaphorically in many aspects of the organizational dynamics of our educational institutions. The use of metaphors in lived experience is described, the scientific background behind strange attractors is introduced, and connections are made between strange attractors and human interaction. Strange attractors are then metaphorically described in organizational settings as shared vision, team processes, and information flows used as positive feedback mechanisms.
\end{abstract}




\section{Introduction}

A number of systems and leadership theories have arisen during the twentieth century, ranging from scientific management to business reengineering. Yet historical and contemporary management strategies have typically incorporated models that rely on classical mechanics as a basis for application. Perhaps the most recognizable form of organizational transition in this modernistic framework is one in which managers introduce corrective measures to move an organization slowly and incrementally towards future goals (Newman, 2000). In effect, external and internal stimuli are significantly controlled to keep the dissipation of energy low while the organization moves towards relatively stable equilibrium (Stacey, 1992b; 2003). We commonly understand this type of leadership phenomena through the metaphor, "keeping the ship on course." However, in educational settings this framework sometimes perpetuates the problematic aspects of leadership.

Granted, we might all like to work, teach, and lead in a near-equilibrium environment. At face value, this system appears to exhibit less stress and certainly limits the ability of external forces that dictate changes in our educational institutions. But this type of setting does not quite seem to exist in North American schools and colleges. As educators, we are confronted with a changing student body with diverse educational needs. We try to balance quality teaching with the external pressures of accountability and high-stakes testing. We are constantly challenged by leaders in the business community who argue schools should be customer-driven but who do not realize that in this theoretical framework our students are equally producers and products (Birnbaum, 2000). When national economic downturns take place, our schools and colleges are confronted with tough decisions on how to cut budgets in order to meet the bottom line. Furthermore, leaders at the highest levels feel that power is restricted in the practical application of management regardless of the approach (Morgan, 1997). We see more frequently the paradox of conflicting external and internal needs at the micro and macro levels that severely limit our ability to maintain order and move our schools towards equilibrium.

In effect, we are operating in chaotic systems where organizational structures are challenged in ways we might never have predicted. However, "if you see reality as defined by the metaphor ... then you can answer the question relative to whether the metaphorical entailments fit reality" (Lakoff \& Johnson 2003, p. 158). Prigogine and Stengers (1984) have shown that dynamical systems are actually more closely aligned with the natural world. Leadership and educational researchers equally argue there is tremendous potential for the metaphorical significance of complexity science in organizational dynamics (Doll, 1993; Fleener, 2002; Fullan, 2001; Karpiak, 2000; Mor- 
gan, 1997; Senge, 2004; Stacey, 2003; Wheatley, 1992). An understanding of chaos metaphors, therefore, might help us navigate through our complex educational environments and provide us with empowering answers to the paradoxes of complex adaptive systems and our approaches to leadership. Although this paper focuses on educational leadership, it is inferred that these same concepts and themes can be transferred to classroom management and pedagogical ontology. It is the purpose of this article, therefore, to demonstrate how the strange attractor metaphor can lend to a further understanding of educational leadership and teaching through shared vision, team processes, and information flows used as positive feedback mechanisms.

\section{Strange Attractors and Metaphors}

\section{Education is in Search of Chaos Metaphors}

To some, the idea of metaphor might be a novelty; one that elicits creativity, amusement, or description but that has no practical application in educational thought. To others, the idea of metaphor might be reserved only for literature or even as a pejorative to describe misguided educational research. However, metaphors truly encompass our everyday communication and thinking patterns, argue linguists Lakoff and Johnson (2003):

In all aspects of life, not just politics or in love, we define our reality in terms of metaphors and then proceed to act on the basis of the metaphors. We draw inferences, set goals, make commitments, and execute plans, all on the basis of how we in part structure our experience, consciously and unconsciously, by means of metaphor. (p. 158)

Placing this language within the framework of both complexity and chaos theory, we can see how powerful and pervasive metaphors really can be in describing and understanding human experience. The confines of this paper do not allow for a comprehensive exploration of scientific thought, but a few common ideas will be defined throughout this article as a method for connecting metaphors with complexity science. Equally, I will borrow from Stacey's (2000; 2003) working definitions to convey meaning of particular subsets of the "new sciences." The term "complexity science" will be used to refer to the umbrella of theoretical thought encompassing all subsequent theories. Chaos theory will be used to describe nonlinear, chaotic systems that are homogeneous in nature and tend to move toward strange attractors. Complexity theory will refer to heterogeneous complex adaptive systems that move toward one or more attractor patterns (Stacey, 2003) and contain the ability for what Osberg and Biesta (2004) describe as "strong emergence," where "what emerges is always radically novel" (Osberg \& Biesta, 2004, p. 210). I will also tend to refer to organizational units as cha- 
otic systems and the educational institution as a complex adaptive system. However, just as quantum theory has shown us wave-particle duality, there will be examples in this paper where it becomes obvious that chaotic systems can contain elements of and exist concurrently and pluralistically as complex adaptive systems.

Returning to complexity science metaphors, there are obvious differences between near-equilibrium and complex adaptive systems. Equilibrium-oriented systems are ones that attempt to control disorder in a desire to move towards a stable state. Because they limit their ability to consume external energy, near-equilibrium systems must behave as closed systems to conserve energy. When foreign stimuli are introduced, the system applies dampening mechanisms (negative feedback) that minimize these externalized effects, allowing the system to return to stability. Conversely, complex adaptive systems contain simultaneous order and disorder. They require exchanges of outside energy that push systems toward bifurcation points in which there is a split. At this moment, it is possible for the system to re-emerge at a higher and more complex level of development-it is transformed.

When we apply the use of scientific systems metaphors in educational settings, we see similarities to different organizational environments. The near-equilibrium educational institution is one that limits the effects of foreign constructs and perturbation, applying negative feedback mechanisms to continue in a comfortable and well-known state. Control mechanisms are firmly in place to preserve order, oftentimes leading to strict policies, rigid hierarchies, resistance to change, and maintenance of the status quo. Conversely, far from equilibrium educational settings are ones that are influenced by several external and internal forces; hierarchical lines begin to become transparent, and the environment becomes attuned to the emergence of the bottom-up, self-organizing principles of dissipative structures. In the areas of leadership and teaching theory, a review of attractor metaphors can lead to a better understanding of this emergent environment.

\section{The Science and Metaphor of Attractors}

Attractors are pervasive in both near-equilibrium and chaotic systems in the scientific world. They act as magnetic forces that draw complex adaptive systems towards given trajectories (Pascale, Millemann, \& Gioja., 2000; Wheatley, 1994). Attractors usually can be defined in four different forms: point, periodic point, periodic, and strange. An exploration of each of these attractor types will show their potential for application as metaphors within organizational dynamics.

The most basic attractor is the point attractor. This attractor can be described as operating in a phase space that moves towards a highly equilib- 
rium state. They "lure systems to a stable position of rest" (Pascale et al., 2000, p. 70). As an example, when a book is dropped, reserved kinetic energy is expelled when the force of gravity pulls the book to the floor; in this case the "point." Once the book reaches this point, it comes to rest in an equilibrium state. At the micro level, point attractors serve as metaphors for limited task completion in educational settings. You have 20 copies of a syllabus to photocopy before attending your first class of the semester. Once completed, the task is finished. At the macro-level, monopolistic organizations are metaphors for point attractors. A university has gained ground over time and now dominates a geographic and technological market share. Consequently, it has no worries about competition from other institutions, so it can now rest on its laurels.

The next attractor is the periodic point attractor. This type of attractor is one that usually moves in a linear or orbital pattern toward and away from a set point a given number of times. Examples of periodic point attractors in the mechanical world would include a gear or a piston in a machine where the object moves in a circular or linear fashion, returning consecutively to the same point with no deviation in its trajectory. This type of attractor is driven towards a stable equilibrium state, as it follows the same path repeatedly until it eventually comes to rest. In an educational setting, the periodic point attractor serves as a metaphor for a repeated event. Expanding on the previous examples of point attractors at the micro level, the task of photocopying syllabi for a particular class may be something that is repeated every year. At the macro level, although a university may not fear market competition, it still disseminates its marketing materials annually to maintain its monopolistic position.

Slightly more complex is the periodic attractor. Similar to the periodic point attractor, the periodic attractor follows an orbital or linear trajectory towards a set point, yet the trajectory of the object can change from iteration to iteration. They "move systems into loops of predictable but dynamic patterns" (Pascale et al., 2000, p. 70). A scientific example of this might be a planet orbiting around a sun-where the trajectory of the planet changes slightly with each revolution-continually returning to its attractor. Periodic attractors also are considered to operate in equilibrium-oriented systems, as their patterns exist in bounded stability. A metaphor for periodic attractors at the micro level might be the editing of a syllabus for a class. Each year, the syllabus must be modified slightly to account for time and classroom changes, yet the content of the syllabus remains relatively the same. At the macro level, departmental budget cycles might serve as a metaphor for a periodic attractor. Each year, the chief financial officer of the institution requests that departments turn in budget proposals, using a timeline and defined criteria for development of the proposal. The timeline and criteria 
might change slightly from year to year, but ultimately, the department chair, in coordination with faculty, develops the budget based both on budget needs at that time and the knowledge that this process will be repeated yearly.

The most complex and chaotic of the lot is the strange attractor. Systems operating within a strange attractor framework move in chaotic patterns of bounded instability. In this way, parameters provide a boundary from which the system does not stray; yet the object's movement within those parameters cannot be predicted within the framework of time or space. "Strange attractors are reflected in patterns of behavior, that is, shapes in space or movements over time, which are never exactly repeated but are always similar to each other" (Stacey, 2003, p. 44). They exhibit low-dimensional chaos, are fractal, and continually fold in on themselves (Stacey, 1996; Williams, 1997). A strange attractor requires high energy and information consumption, and it serves as a seemingly magnetic force that continually draws the system near it during each iterative loop in the system cycle (Stacey, 2003; Wheatley, 1994). The chaotic system cannot be predicted, as all elements do not arrive at the exact point of the strange attractor; rather the system's trajectory folds towards the vicinity of where the strange attractor appears to reside at a given iteration.

Perhaps the most commonly recognized strange attractor is the Lorenz attractor [see figs. $1 \& 2$ ], but there are several other strange attractors, such as the Ikeda and Duffing attractors [see figs. 3 and \& 4] that show visually the dynamics of attractor patterns (Elert, 1995). Represented in three dimensions, the strange attractor is one that continues to fold into itself where a new form emerges with each iteration. The next section will explore the metaphor of the strange attractor in more detail within the educational leadership framework.
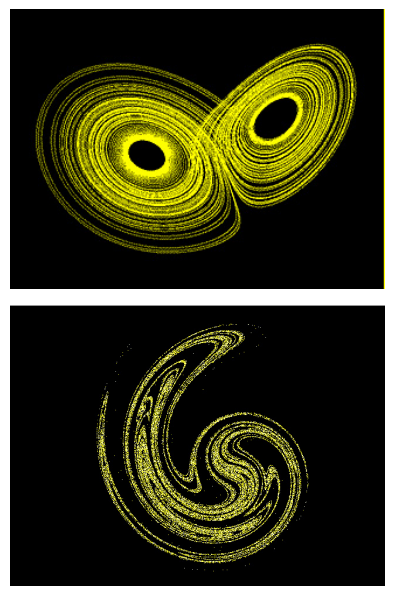

Figure 1

Lorenz

Figure 3

Ikeda
Figure 2

Lorenz
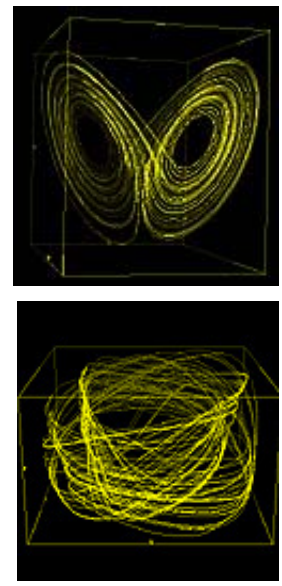

[Fair use of the Chaos Hypertextbook is encouraged; http: / / hypertextbook.com/ contact.shtml] 


\section{Strange Attractors and Human Interaction}

At the macro level, it is difficult to determine if a comprehensive model for complexity science can be applied directly in higher education leadership, since complex systems are unpredictable except for the very short-term. Furthermore, at the micro level, Stacey $(2003$, p. 46) argues, complexity theory "cannot be applied directly to human action because human interaction is not deterministic," yet there can still be control parameters such as energy and information flows. Constas (1998) also reminds us that, although we embrace the pluralism of complexity science, we need to find ways that help bridge the divide between pure and applied aspects of theory in order to find pragmatic methods for utilizing this knowledge in educational settings. Case studies in systems theory have alluded to different areas of complexity science emerging through the application of management techniques at both the micro and macro levels (Breu \& Benwell, 1999; Nadler, Shaw, \& Walton, 1995; Newman, 2000). Equally, case studies by complexity science theorists have shown there are organizational situations where chaos, complexity, and dissipative structures theory can be applied and observed (MacIntosh \& MacLean, 1999; 2001; Pascale et al., 2000).

Complexity science offers a new way to view the world, but for those of us in education, the questions seem to remain: What do we do with this knowledge and how do we move toward a new organizational philosophy of complexity? In this aspect, the mere knowledge of complexity science by students, teachers, faculty, and administrators can serve as a strange attractor in educational settings. Morgan (1997) identifies the creation of the environment where chaos theory can emerge as perhaps the most important contribution educational leaders can provide for their institutions. Chaos theory "encourages us to develop mind-sets and skills that focus on recognizing and changing patterns.... It provides a methodology for analyzing a system's 'attractor patterns' and for changing the trajectory" (p. 282). Interestingly, the strange attractor is the most common attractor in natural systems, yet oftentimes these attractor patterns exist competitively between point, periodic, and strange attractors; or, as Morgan (1997, p. 271) argues, "between the status quo and alternative future states."

These are obviously difficult challenges for teachers and educational administrators. As we have seen in Prigogine $(1967 ; 1980 ; 1997)$ and others' works, the pull towards equilibrium attractors is so strong that, unless the system exists in an environment ready for change, it will return to equilibrium (Doll, 1993; Fleener, 2002; Gemmil \& Smith, 1985; Karpiak, 2000; MacIntosh \& MacLean, 2001; Nicolis \& Prigogine, 1989; O'Sullivan, 1999; Pascale et al., 2000; Prigogine, 1980; 1997; Prigogine \& Stengers, 1984). However, the concept of shared vision as a strange attractor metaphor can possibly lead toward that environment of change. 


\section{Strange Attractors and Organizational Dynamics}

As we move away from linear determinism in educational planning, we are confronted with ambiguous perceptions of how to implement methods that will allow for emergent complex adaptive systems. Educational leaders might argue that vision provides a road map to a specific future, and that they know how to implement the necessary actions that will lead the institution to that point. In reality, the concept of vision should be dynamic and fluid in organizational settings, as leadership successes are often realized through listening to employees and through discovery (Stacey, 1992b).

When we view the strange attractor metaphor, Lakoff and Johnson (2003) remind us of the dualism of position power metaphors bring us:

Most of our metaphors have evolved in our culture over a long period, but many are imposed upon us by people in power.... In a culture where the myth of objectivism is very much alive and truth is always absolute truth, the people who get to impose their metaphors on the culture get to define what we consider to be true. (pp. 159-60)

As a result, dictating the metaphor of the strange attractor will most likely lead to outcomes that are anathema to desired effects. In the same way, shared vision as a strange attractor metaphor is something that cannot be mandated or introduced by leaders; it must emerge from within the organization based on the experiences of the people working there (Fullan, 2001; Morgan, 1997; Pascale et al., 2000; Stacey, 1992b). If that is the case, then we can see how energy and resources are currently diverted towards wasteful, top-down processes that, from the outside, appear to be models for successful achievement. And given Morgan's (1997) observation on the restrictions of power in relation to these processes, we can imply leaders are inadvertently allowing self-organizing principles to take hold on the inside anyway.

\section{Shared Vision as an Alternative to Traditional Strategic Planning}

In the complex environment, traditional models of strategic planning become less reliable methods for helping the future emerge from within the present. The very concept of goal setting is deterministic - a linear path towards desired, predictive outcomes. Traditional strategic planning processes are usually linked with action plans, yet, causal relationships disappear within a complexity framework, dampening our ability to determine the actions necessary to complete specified goals (Pascale et al., 2000; Stacey, $1992 b)$. Returning to the attractor metaphors, goal setting activities can become too deterministic and rigid, leading educational systems towards point or periodic point attractors where the outcome of the process is to reach a certain point upon a linear trajectory or similar to what Osberg and Biesta (2004) describe as "weak emergence." Once the process is completed, it is 
either treated as task completion or preparation begins anew for the coming cycle of the same process. Contrast this with the strange attractor which has an "irregular, erratic, trajectory" with "a total absence of crossing trajectories" (Williams, 1997, p. 228), and we can easily see that the point and periodic attractor metaphors of goal setting activities drive educational institutions towards equilibrium.

Cutright (2001) equally argues against traditional forms of strategic planning where the linear nature of top down directives leads to insincere initiatives by lower levels of staff. Moreover, "attractors are not identified, feedback is denied, faint recognition of the environment is inevitable, and the implementation of plans is made virtually impossible by the lack of fractal structure" (p. 67). It is from our knowledge of the incapacity of these linear models to which we are accustomed that we must search for new meaning in the process. Shared vision is not top-down, directive, or enforced (Morgan, 1997), as these factors would lead to a subjugated or single vision. The use of shared vision does not see the present as a controlling variable for the future (Pascale et al., 2000, p. 71), rather, it acts as a frame of reference where the future is unfolded within the dynamics of the organization. Wheatley (1994) describes shared vision through field theory, where if the vision truly exists, it's as though people "who bumped up against the field would be influenced by it" (p. 54). Shared vision through a complexity lens subsequently challenges so many of our basic beliefs in teaching and leadership and confronts us with the dilemma of how to incorporate shared vision into organizational settings without directing it.

Morgan (1997) contends the primary responsibility of leaders is to create the environment where the elements of complexity science can emerge. As an educational leader, merely presenting the issues of the current situation and possible scenarios for the future can lead to the emergence of shared vision as a strange attractor, since it provides an opportunity for everyone in the organization to give individual meaning to what they are hearing. As an example, when Robert Shapiro took over as CEO of the petrochemical company Monsato in 1993, he was confronted with a challenging new biotechnology environment that could quickly lead to the demise of the company. Shapiro led town hall meetings where he discussed his observations of the problems with which the company was confronted and offered different directions the company might need to move in the future in order to deal with the changing environment (Pascale et al., 2000). Shapiro didn't provide the answers, and, consequently, employees came away from the meetings critically reflecting on their own interpretations of what the problems were while self-organization phenomena began to take place. Equally, Ford Motor Company's success in the 1990s came from the company motto, "Quality is Job 1" (Pascale et al., 2000), where employees' shared vision of quality became an encompassing aspect of each individual's work. 
Contrast these cases with "IBM Means Service" which evolved from a shared vision to a single vision. The information technology environment had changed drastically, yet IBM employees still strove towards this mantra since it represented a comfort level with which they were familiar. Subsequently, the organization became a closed system, separating itself from the changing external environment (Morgan, 1997) and losing its domination of the mainframe industry. Employees at both Monsato and Ford, however, realized they were involved in something bigger and better than themselves, and this shared vision became "a means of giving expression to a life purpose" (Pascale et al., 2000, p. 245). Equally, taken as groups within the larger organization, these companies became complex adaptive systems while internal units began operating as chaotic systems.

Returning to the strange attractor metaphor, if every person in an educational setting shares a single vision through traditional strategic planning models, then, by definition, this single vision is operating as a point or periodic attractor, leading the organization toward equilibrium. Teachers and educational leaders can help to create the parameters for the attractor, but it is the internal dynamic rules that lead to the emergence of new attractors. Shared vision, subsequently, must be a fluid and continually changing process where individuals recognize the vision of the institution and move iteratively towards it as a strange attractor. Yet there is still room for views that conflict with that vision or that can change the vision as each iterative loop in the creative planning process unfolds with new order (Stacey, 1996). Most importantly, in order to function as a strange attractor, people must continually question the purpose of the shared vision (Stacey, 1992b). The metaphoric attributes of the strange attractor then emerge, as the shared vision for the institution changes over time and space, folds in on itself, and develops new, higher-level order. Shared vision, therefore, acts as a strange attractor in a chaotic system and can iteratively lead the school to become a complex adaptive system.

\section{Emerging Attractors through Team Processes}

Shapiro's approach to shared vision at Monsato also serves as a view for teachers and educational leaders to incorporate the strange attractor metaphor through team processes. Rather than providing the answers for Monsato employees, Shapiro simply stated the problems and provided possible scenarios for the future. These meetings were interpreted in different ways by company employees who were able to process what they believed to be hearing based on their own knowledge and experiences in the company. Prigogine $(1980,1997)$ and others describe this process as self-referencing, where a system utilizes existing information, or initial conditions, 
in the process leading toward a bifurcation point. (Breu \& Benwell, 1999; Fleener, 2002; Fullan, 2001; Gemmil \& Smith, 1985; MacIntosh \& MacLean, 1999, 2001; Nicolis \& Prigogine, 1989; O’Sullivan, 1999; Pascale et al., 2000; Prigogine \& Stengers, 1984).

Team-based environments are essential to complex organizations, since human dynamics tend to follow patterns that more closely align with the natural elements of chaotic systems, such as self-referencing and self-organization. In the complex organizational environment, Van Olffen \& Romme's (1995) and Smith \& Comer's (1994) research shows that teams can process information at a fast rate, that creativity spontaneously emerges in team settings, that teams can handle and adapt to turbulence and ambiguity better, and that "effective teams are units acting according to the image of dissipative self-organization" (Van Olffen \& Romme, 1995, p. 204). Since they are much less structured than many rigid organizational hierarchies, teams are open to outside influences and rely on increased information flows (open systems), their responsibilities are more loosely defined and cross over into different areas (nonlinear), they share knowledge and have redundancy built into the system (self-referencing), they tend to self-select members (organic), and they usually embrace a shared vision, culture, or meaning (strange attractor) (Fullan, 2001; Morgan, 1997).

Another benefit of teams is their ability to reprocess information from the individual to the organizational level. After individual reflection on the problems facing an organization, the complexity dynamic of strong emergence can take place. Individuals return to teams to discuss what they have heard. Redundancy built into team-structures helps people to focus on the same problems and seek scenarios that may be synthesized or that actually compete. However, the dynamics of each team lead to group interaction where individuals subsequently generate pluralistic views of individual experiences. Members of teams then connect with other teams and the process continues recursively. In this way, individuals in teams can embrace and find connectedness in the plurality of diverse views (Doll, 1993). Equally, it is apparent how critical the bottom-up method of team interaction is to strong emergence in organizational transformation.

\section{Information Flows as Positive Feedback Mechanisms}

Feedback mechanisms are an integral part of the strange attractor process, as they help to construct the parameters of a system. Moreover, they can be used to facilitate the creation of simultaneous strange attractors - which leads by definition to the emergence of a complex adaptive system (Stacey, 2003). However, it must be noted that overuse of feedback controls ultimately pushes a system towards equilibrium (Eisner, 1981; Stacey, 1992a; 1992b; 1996). This 
being the case, micromanagement techniques that attempt to control continuously the direction of an educational organization are anathema to the emergent properties of complex systems. Furthermore, negative feedback has a tendency to return a system to equilibrium (Pascale et al., 2000; Stacey, 1992b; 2003). Albeit, there are times when teachers and educational leaders have both moral and professional obligations to apply negative feedback, such as when employees or students are acting in unethical ways or when such rigid constraints have been placed on a school or college by external forces that the organization must move towards a stable attractor. However, some leadership techniques, particularly positive feedback mechanisms, can be introduced occasionally into an organization in order to help maintain the system's parameters or create new attractors as stated previously.

Information flows are successful components of positive feedback. Wheatley (1994) suggests that flows of information within a system foster self-organization, and Stacey $(1992 b ; 1996 ; 2003)$ sees the speed of these information flows as control mechanisms for creating system parameters. Since the introduction of new ideas within an educational institution oftentimes leads to competition with the equilibrium driving forces that attempt to return the organization to a stable state, educators and administrators can subsequently focus information flows towards an emerging strange attractor. These information flows can act as the high amounts of energy a dissipative system needs to consume to move closer to bifurcation.

In educational settings, multiple information flows serve as a critical component of chaotic systems, leading to complex adaptive systems. In the nonlinear educational organization, the number of connections between individuals determines the level of complexity of the school. If connections between people are few, then the system will move towards a stable attractor pattern (Pascale et al., 2000). But since chaotic systems require high consumption of information, increasing the connections between people can serve as a strange attractor. Returning to the concept of teams discussed previously, educational leaders can apply positive feedback mechanisms that increase the amount of information flows within and among teams, furthering connectivity. Subsequently, this influx of external energy can help the self-organization process through continual self-referencing and reanalysis of problems, leading to an increase in the amount emerging strange attractors.

\section{Conclusion:}

\section{Metaphor and Strange Attractors in Educational Settings}

It is safe to assume most chaos and complexity theorists agree that any application of a model with rigid parameters would not fit into a complex adaptive system and that the over-application or micromanagement of feed- 
back mechanisms to control a complex system would drive it towards equilibrium. Moreover, highly controlled social processes lead humans towards equilibrium; the more control, the more systems move away from strange attractors toward periodic and point attractors. Although Stacey (2003) continues to argue against the application of complexity theory at the macro level, he suggests that our use of strange attractor metaphors can coincide with metaphors describing human interaction. Subsequently, strange attractors can serve as chaos metaphors that lead to strong emergence in complex adaptive systems.

This article provides methods for identifying and using strange attractor metaphors to facilitate emergent, complex educational environments. Shared vision can serve as a strange attractor metaphor that iteratively moves people in an educational institution towards a relative and dynamic cultural philosophy. At the same time, this attractor framework enables individuals simultaneously to retain and transform their own identities within and perceptions of the organization. As a result, team processes can emerge as another strange attractor metaphor, where the principles of self-organization foster recursive planning processes and continual re-generation of the shared vision attractor in educational settings. And, finally, information flows can be used as positive feedback mechanisms that increase the rate of organizational energy consumption. Over time, these information flows might even emerge as strange attractors, because individuals move toward the flow of information to continue high energy dissipation within team interactions. If we embrace the power of each of the strange attractor metaphors presented in this article, we can transform our understanding and description of educational leadership and pedagogical ontology in ways that more accurately convey the environments within which we teach and lead.

\section{Acknowledgements}

This article is a modified draft of a manuscript originally presented at the American Educational Research Association's 2005 Annual Meeting in Montréal, Québec, for the Chaos and Complexity SIG. My thanks go out to the members of the Chaos and Complexity SIG and to the Complicity editorial board and reviewers for their insightful feedback that has led this work to its present stage.

\section{References}

Birnbaum, Robert. 2000. Management fads in higher education: Where they come from, what they do, why they fail. San Francisco: Jossey-Bass.

Breu, K., and M. Benwell. 1999. Modelling individual transition in the context of organisational transformation. Journal of Management Development 18(6): 496-520.

Constas, Mark A. 1998. The changing nature of educational research and a critique of postmodernism. Educational Researcher 27(2): 26-33. 
Cutright, Marcus. 2001. Chaos theory E higher education: Leadership, planning, E policy. New York: Peter Lang.

Doll, William E., Jr. 1993. A post-modern perspective on curriculum. New York: Teachers College Press.

Doll, William E., Jr. 1987. Foundations for a post-modern curriculum. Paper presented at the Annual Meeting of the American Educational Research Association, Washington, DC. April 20-24.

Eisner, Eliot W. 1981. On the difference between scientific and artistic approaches to qualitative research. Educational Researcher 10(4): 5-9.

Elert, Glen. 1995. Chaos hyptertextbook: Mathematics in the age of the computer. http:/ / hypertextbook.com (accessed September 1, 2005).

Fleener, M. Jayne. 2002. Curriculum dynamics: Recreating heart. New York: Peter Lang.

Fullan, Michael. 2001. Leading in a culture of change. San Francisco: CA: Jossey-Bass.

Gemmill, G. and C. Smith. 1985. A dissipative structure model of organization transformation. Human Relations 38(8): 751-766.

Karpiak, Irene. E. 2000. Evolutionary theory and the "new sciences": Rekindling our imagination for transformation. Studies in Continuing Education 22(1): 29-44.

Lakoff, George and Mark Johnson. 2003. Metaphors we live by. Chicago: University of Chicago Press.

MacIntosh, Robert and Donald MacLean. 1999. Conditioned emergence: A dissipative structures approach to transformation. Strategic Management Journal 20(4): 297-316.

MacIntosh, Robert and Donald MacLean. 2001. Conditioned emergence: Researching change and changing research. International Journal of Operations and Production Management 21(10): 1343-1357.

Morgan, Gareth. 1997. Images of organization (second ed.). Thousand Oaks, CA: Sage Publications.

Nadler, D.A., R.B. Shaw, and A.E. Walton. 1995. Discontinuous change: Leading organizational transformation. San Francisco: Jossey-Bass.

Newman, Karen L. 2000. Organizational transformation during institutional upheaval. Academy of Management Review 25(3): 602-619.

Nicolis, Grégoire and Ilya Prigogine. 1989. Exploring complexity: An introduction. New York: W.H. Freeman and Company.

O'Sullivan, Edmund. 1999. Transformative learning: Educational vision for the $21^{\text {st }}$ century. New York: St. Martin's Press.

Osberg, Deborah and Gert J.J. Biesta. 2004. Complexity, knowledge and the incalculable: Epistemological and pedagogical implications of 'strong emergence'. Paper presented at the Complexity Science and Educational Research Conference, Chaffey's Locks, Canada, Sept 29-Oct 1.

Pascale, Richard T., Mark Millemann, and Linda Gioja. (2000). Surfing the edge of chaos: The laws of nature and the new laws of business. New York: Crown Business.

Prigogine, Ilya. 1967. Thermodynamics of irreversible processes. New York: John Wiley \& Sons.

Prigogine, Ilya. 1980. From being to becoming: Time and complexity in the physical sciences. San Francisco: W.H. Freeman and Company.

Prigogine, Ilya. 1997. The end of certainty: Time, chaos, and the new laws of nature. New York: The Free Press.

Prigogine, Ilya. 2000. The future is not given, in society or nature. NPQ: New Perspectives Quarterly 17(2): 35-37. 
Prigogine, Ilya and Isabelle. 1984. Order out of chaos: Man's new dialogue with nature. New York: Bantam Books.

Smith, Charles and Debra Comer. 1994. Self-organization in small groups: A study of group effectiveness within non-equilibrium conditions. Human Relations 47(5): 553581.

Stacey, Ralph D. 1992a. Managing chaos: Dynamic business strategies in an unpredictable world. London: Kogan Page.

Stacey, Ralph D. 1992b. Managing the unknowable: Strategic boundaries between order and chaos in organizations. San Francisco: Jossey-Bass.

Stacey, Ralph D. 1996. Complexity and creativity in organizations. San Francisco, CA: Berrett Koehler.

Stacey, Ralph D. 2003. Complexity and group processes: A radical social understanding of individuals. New York: Brunner-Routledge.

Stacey, Ralph D., Douglas Griffin, and Patricia Shaw. 2000. Complexity and management: Fad or radical challenge to systems thinking? New York: Routledge.

Van Olffen, Woody and A. Georges L. Romme. 1995. The role of hierarchy in self-organizing systems. Human Systems Management 14: 199-206.

Wheatley, Margaret J. 1994. Leadership and the new science: Learning about organization from an orderly universe. San Francisco: Berrett-Koehler Publishers, Inc.

Williams, Garnett P. 1997. Chaos theory tamed. Washington, DC: National Academies Press.

\section{About the Author}

Donald Gilstrap is the Director of Libraries at Southwestern Oklahoma State University, 100 Campus Drive, Weatherford, OK, USA. He holds a B.A. in History and French, an M.A. in History, and an M.L.S. He is a Ph.D. candidate in the University of Oklahoma's Educational Leadership and Policy Studies Program. His current research interests are primarily focused on leadership theory; organizational dynamics; and chaos, complexity, and dissipative structures theories. He can be reached by telephone at 580.774 .7081 , and his email address is donald.gilstrap@swosu.edu.

(C) Copyright 2005. The author, Donald L. Gilstrap, assigns to the University of Alberta and other educational and non-profit institutions a non-exclusive license to use this document for personal use and in courses of instruction provided that the article is used in full and this copyright statement is reproduced. The author also grants a non-exclusive license to the University of Alberta to publish this document in full on the World Wide Web, and for the document to be published on mirrors on the World Wide Web. Any other usage is prohibited without the express permission of the author. 
